\title{
User-Friendly End Station at the ALS \\ for Nanostructure Characterization
}

\section{Final Report}

\section{F. J. Himpsel, University of Wisconsin Madison}

Co-PIs: P. Alivisatos (UC Berkeley), T. Callcott (UT Knoxville), J. Carlisle (ANL), J. D. Denlinger (LBNL), D. E. Eastman (University of Chicago), D. Ederer (Tulane University), Z. Hussain (LBNL), L.J. Terminello (LLNL), T. Van Buuren (LLNL), R. S. Williams (Hewlett Packard Palo Alto).

Additional collaborations with:

A. T. Young (ALS, LBNL)

C. T. Black and C. B. Murray (IBM Yorktown Heights)

D. Y. Petrovykh, J. M. Sullivan, L. J. Whitman (Naval Research Lab)

H. Kimura-Suda, A. Opdahl, M. J. Tarlov (NIST Gaithersburg)

Nicholas L. Abbott, Yan-Yeung Luk, Paul Nealey (Chem. Engineering, UW-Madison)

P. Hoffmann, D. Schmeisser, H.-J. Engelmann, E. Zschech, H. Stegmann (Techn. U. Cottbus and Zeiss, Germany)

\section{Overview}

This is a construction project for an end station at the ALS, which is optimized for measuring NEXAFS of nanostructures with fluorescence detection. Compared to the usual electron yield detection, fluorescence is able to probe buried structures and is sensitive to dilute species, such as nanostructures supported on a substrate. Since the quantum yield for fluorescence is $10^{-4}-10^{-5}$ times smaller than for electrons in the soft $\mathrm{x}$ ray regime, such an end station requires bright undulator beamlines at the ALS. In order to optimize the setup for a wide range of applications, two end stations were built:

1) A simple, mobile chamber with efficient photon detection $\left(>10^{4}\right.$ times the solid angle collection of fluorescence spectrographs) and a built-in magnet for MCD measurements at EPU beamlines (Fig. 1 left). It allows rapid mapping the electronic states of nanostructures (nanocrystals, nanowires, tailored magnetic materials, buried 
interfaces, biologically-functionalized surfaces). It was used with BL 8.0 (linear polarized undulator) and BL 4.0 (variable polarization).

2) A sophisticated, stationary end station operating at Beamline 8.0 (Fig. 1 right). It contains an array of surface characterization instruments and a micro-focus capability for scanning across graded samples (wedges for thickness variation, stoichiometry gradients, and general variations of the sample preparation conditions for optimizing nanostructures).

Both end stations are aimed at providing a rapid feedback loop between synthesis of nanostructures and their characterization (particularly the electronic states). The Molecular Foundry at Berkeley will provide a great opportunity for such feedback.

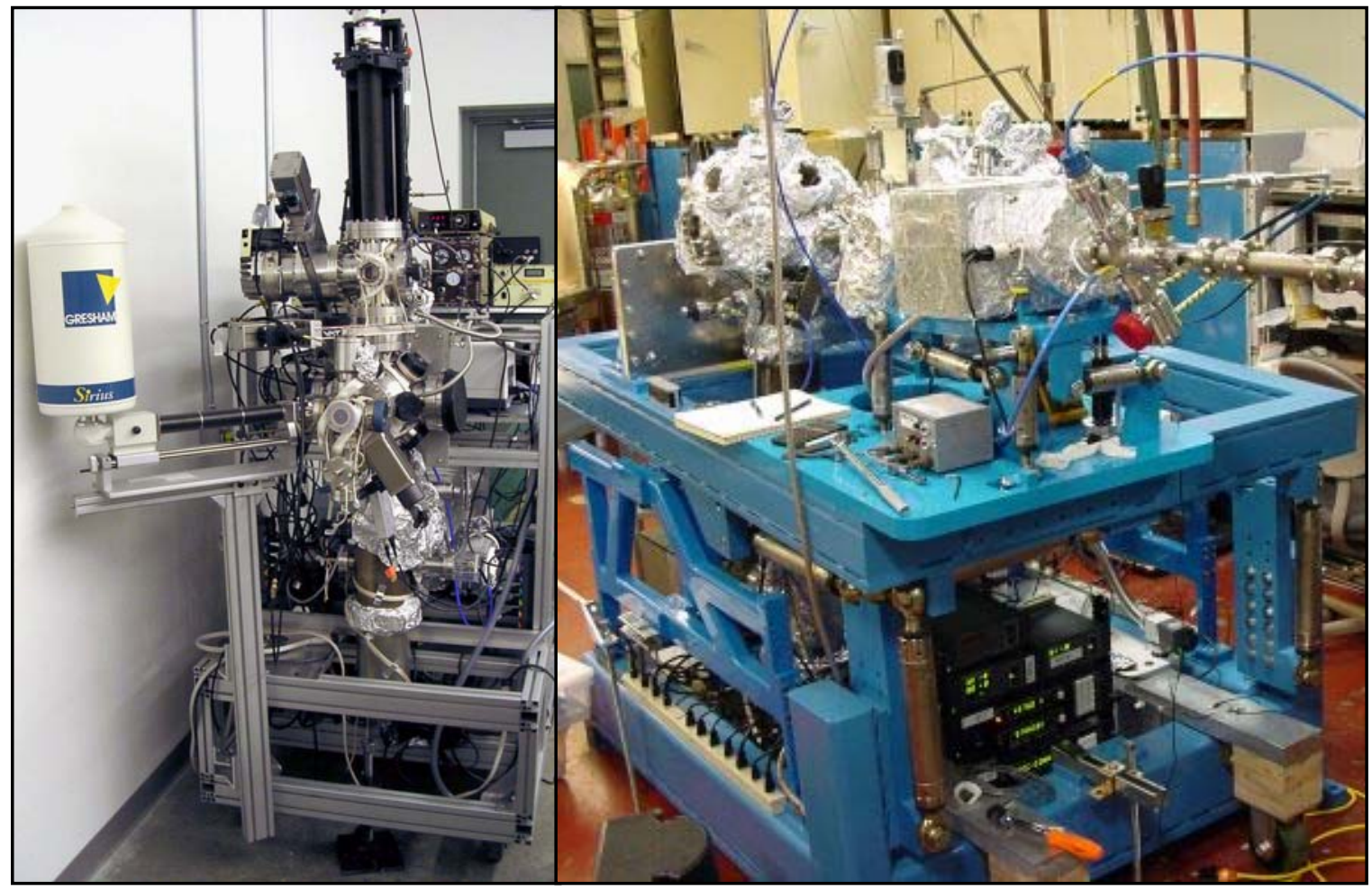

Figure 1: The two end stations built for this project.

The funds were completely committed by spring 2006 (see a separate file with the financial statement). Both chambers are operational and have produced data for 13 publications (listed at the end). There is one remaining task, i.e., assembly work on the mirror tanks and $\mathrm{KB}$ focusing mirrors that reconfigure Beamline 8.0 such that the large end station 2) becomes stationary. This is essential for rapid a turn-around time and an 
efficient feedback loop with synthesis. ALS staff has been assembling the components, and the plan is to install the new switching system during the 2006 fall shutdown.

Personnel: Virginia Perez-Dieste, a postdoc with experience in user support at synchrotron light sources, was supported by the project for two years. She is now scientist at the Swiss Light Source.

Publications: Although this is a construction project, it has produced 13 publications already. Among them is an ALS highlight (publication Nr. 10 below): http://www-als.lbl.gov/als/science/sci archive/128dna.html

\section{Highlights of the Results}

\section{DNA and Protein Immobilization}

Immobilization of DNA and proteins at surfaces is a fast-growing area of research aimed at the development of solid state biosensors that allow rapid testing in the field. First, an inorganic surface, such as silicon or glass needs to be made biocompatible by a sequence of self-assembled monolayers. Typically, one starts with an alkanethiol passivation layer and attaches specific end groups to some of the alkane chains that connect to ever larger organic molecules. The outermost layer consists of the active groups, which can be either single-stranded DNA for the recognition of its complementary DNA sequence in bio-chips, or a protein antibody for the recognition of a virus. For the first type of bio-sensor we collaborate with teams at NRL and NIST (Publication 10), for the second type with teams at the University of Wisconsin-Madison (Publication 12).

In both cases, NEXAFS provides two critical pieces of information: The chemical sensitivity of the technique provides element and orbital-specific information about each step in the creation of a biosensor layer. Traditional techniques for analyzing such thin biological layers, such as ellipsometry, attenuated total reflection, and a quartz microbalance are not sensitive to the chemical composition at all. NEXAFS identifies the elements via the choice of the core level, and the chemical bonding via the empty molecular orbital involved in the optical transition. This technique is even capable of determining the orientation of bio-molecules from the polarization dependence of the 
signal. That is crucial for biosensors. Single-stranded DNA on the surface of the bioensor has to present itself to the complementary external DNA that one wants to detect. Likewise, an antibody on the bio-sensor needs to point outwards for docking to a virus.

Figures 2 and 3 show characteristic NEXAFS data for DNA and proteins, respectively. Some of the key orbitals are detected by NEXAFS at the N 1s edge, i.e., the $\pi^{*}$ orbitals of the nucleotide bases in DNA and the $\pi^{*}$ orbitals of the peptide bond linking the amino acids in proteins. In both cases, we have found a measurable polarization dependence. A quantitative analysis provides the orientation of these molecules, which needs to be controlled for an effective bio-sensor.

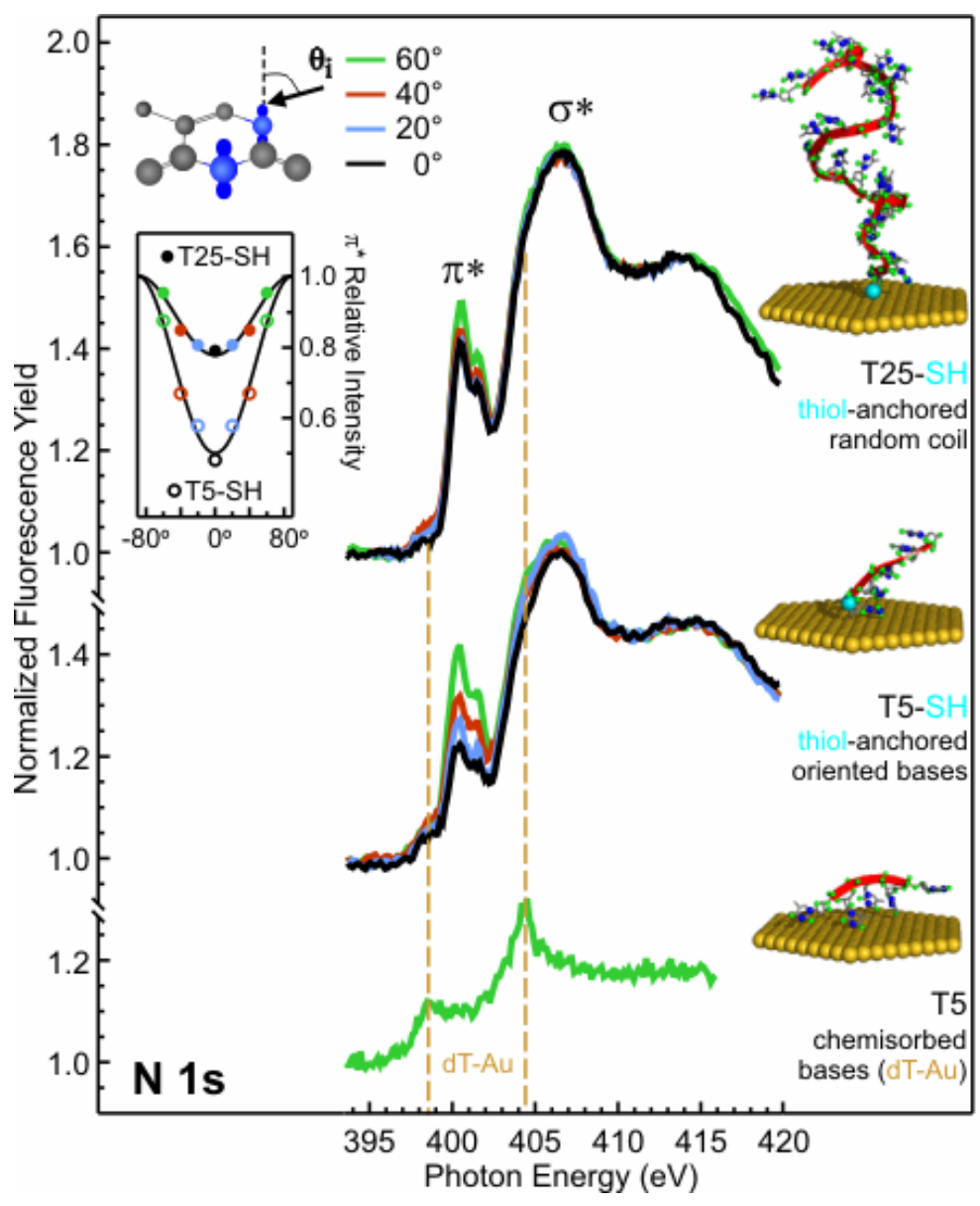

Figure 2: Polarization-dependent NEXAFS of single-stranded DNA. Short segments of 5 thymine bases (T5) show a strong polarization dependence, while longer sections of 25 thymine bases (T25) become more randomly oriented (see the inste for the quantitative polarization dependence). If the surface is not passivated properly, an unwanted direct chemisorption occurs (dT-Au), which is seen as a downward shift of the $\pi^{*}$ and $\sigma^{*}$ orbitals of the bases (dashed lines). From Publication 10. 

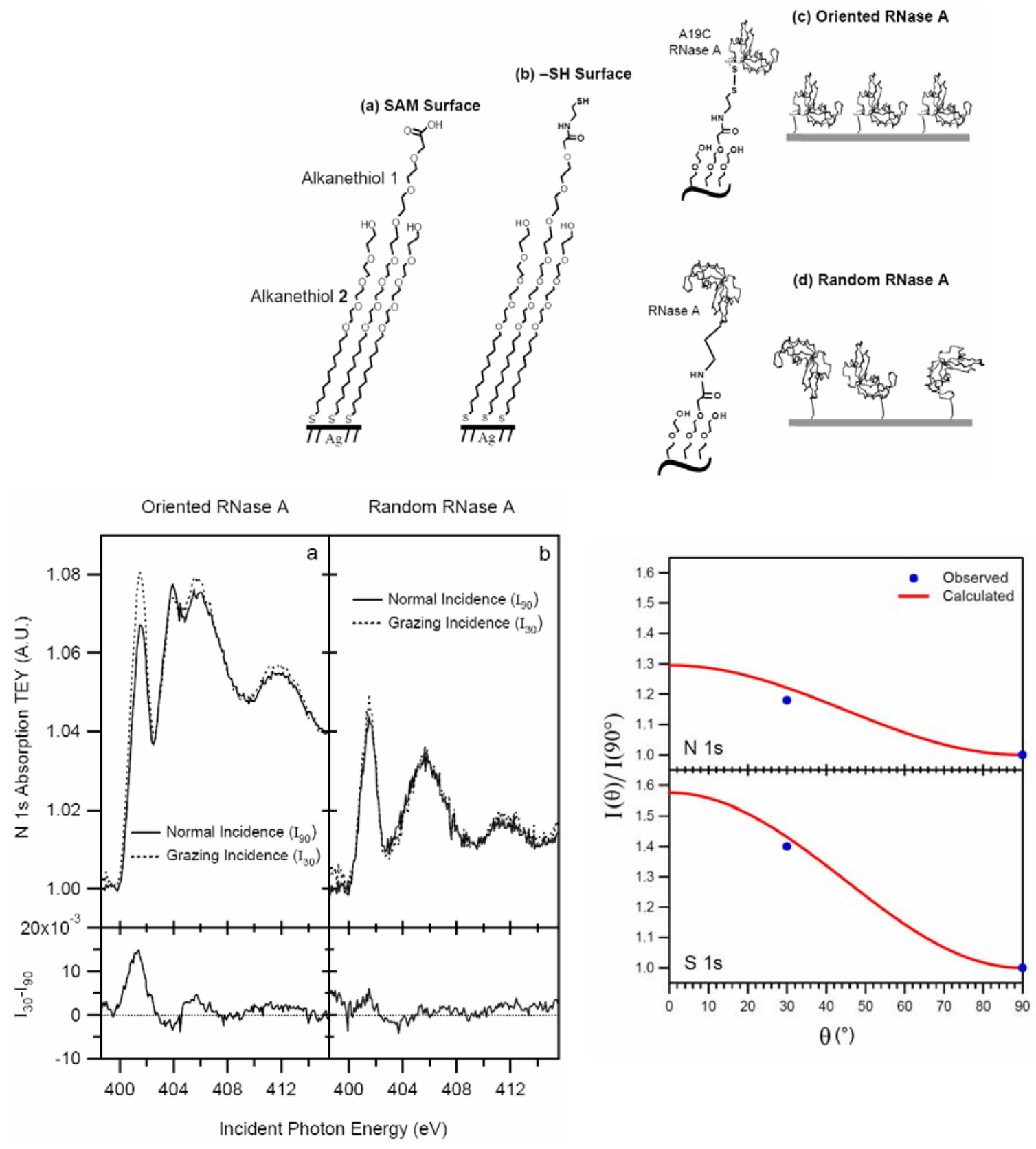

Figure 3: NEXAFS from the protein RNase A (124 amino acids, 951 non-H atoms).

Top: Schematic of the deposition process, showing intermediary layers between the substrate and the protein. Their deposition is monitored step-by-step with NEXAFS.

Bottom left: NEXAFS from the N 1s core level into the $\pi^{*}$ orbital of the peptide bond, which links the amino acids in the protein. For oriented immobilization (c), there is a detectable polarization dependence, which is absent for random immobilization (d).

Bottom right: Experimental polarization dependence for oriented RNase A compared to the polarization dependence calculated from the protein data base without adjustable parameters. For both the $\mathrm{N} \mathrm{1s}$ and the S 1s NEXAFS, there is remarkably good agreement, demonstrating how NEXAFS can be used up to characterize the orientation of biological molecules. From Publication 12. 


\section{Magnetic Nanocrystals with Organic Coating}

Nanocrystals can now be synthesized with nearly atomic precision by relatively simple chemical methods, which opens a wide range of applications encompassing magnetic data storage, non-volatile silicon memory, solar cells, and non-bleaching fluorescent markers in cell biology. We have collaborated with Chris Murray and his group at IBM Research in Yorktown Heights (Publication 5), who pioneered the synthesis of highly-perfect magnetic nanocrystals. These are coated by a protective monolayer of organic molecules. More general, it is becoming clear that the coating of nanocrystals plays a key role in their electronic performance, since a large fraction of the atoms in a nanocrystal are affected by the surface.

In the case of magnetic nanocrystals, thermal cycling is required for obtaining the proper magnetic characteristics. Chemical reactions are induced in the organic coating by thermal cycling, and these could be detected by NEXAFS, as shown in Fig. 4. Comparing NEXAFS in fluorescence and electron detection, one can see the difference between the bulk and the skin of the nanoparticles. The respective probing depths are $100 \mathrm{~nm}$ versus 2 $\mathrm{nm}$, i.e., large and small compared to the $10 \mathrm{~nm}$ diameter of the nanocrystals. Two processes are found, i.e., partial desorption of the surfactant at low temperature $\left(\sim 200{ }^{\circ} \mathrm{C}\right)$ and dehydrogenation at high temperature $\left(\sim 400{ }^{\circ} \mathrm{C}\right)$. The former is evidenced by a decrease of all NEXAFS peaks characteristic of oleic acid, the latter by the loss of the hydrocarbon peaks and the dominance of the $\mathrm{C}=\mathrm{C} \pi^{*}$ peak. 

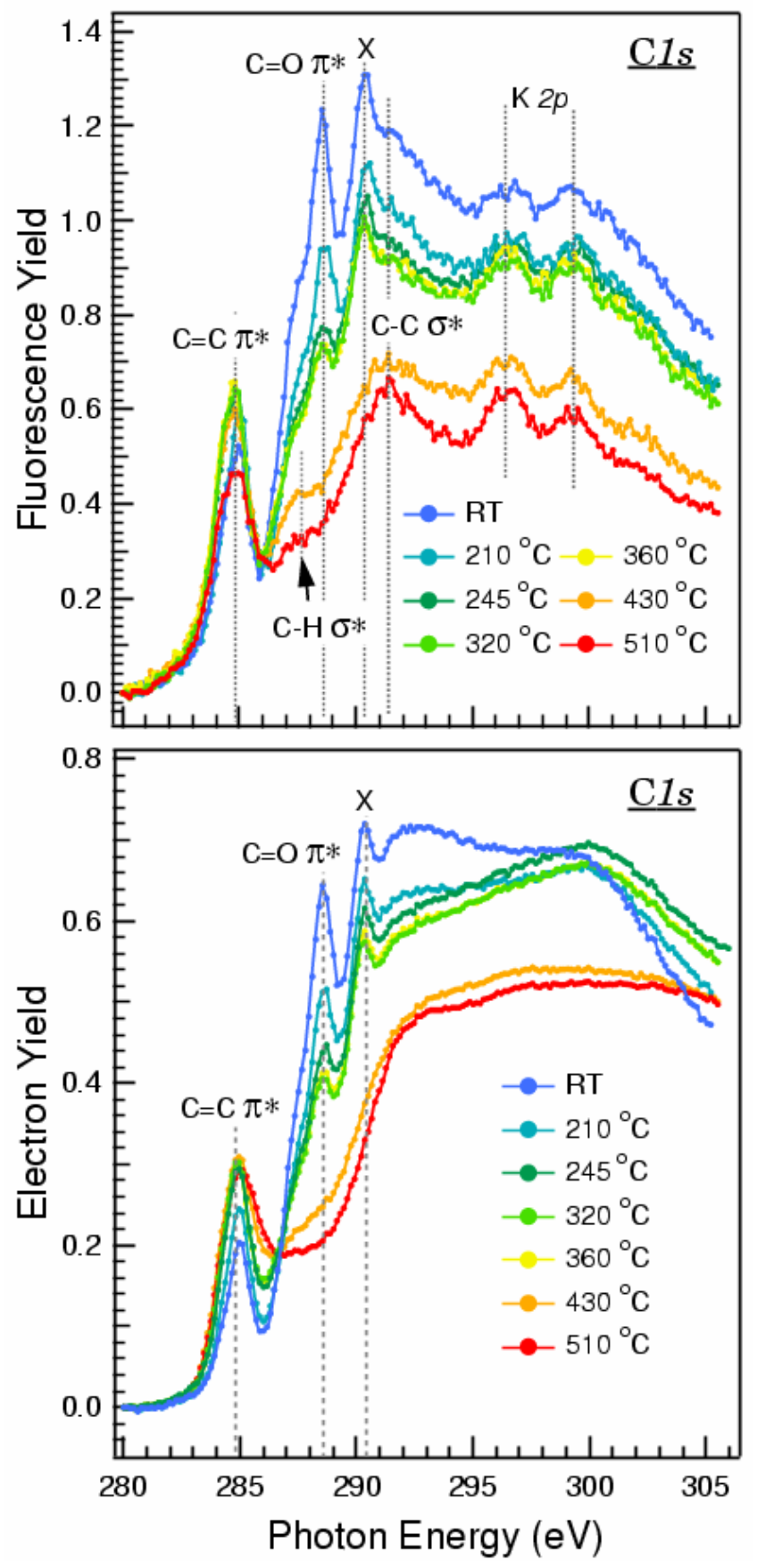

Figure 4: NEXAFS from magnetic nanocrystals during thermal cycling, showing the chemical decomposition of the organic coating. Fluorescence detection (top) doubles the signal/background ratio compared to electron detection (bottom), as shown on the y-axis. It also is able to detect a signal from buried layers, such as the $\mathrm{K} 2 \mathrm{p}$ peaks of a potassium impurity. From Publication 5. 


\section{Publications, DOE Contract No. DE-FG02-01ER45917:}

1. J. L. McChesney, A. Kirakosian, R. Bennewitz, J. N. Crain, J.-L. Lin, and F. J. Himpsel, Gd disilicide nanowires attached to $\mathrm{Si}(111)$ steps, Nanotechnology 13, 545 (2002).

2. J. N. Crain, K. N. Altmann, C. Bromberger, and F. J. Himpsel, Fermi Surfaces of Surface States on Si(111) + Ag, Au, Phys. Rev. B 66, 205302 (2002).

3. F.J. Himpsel, Electronic and Magnetic Materials, in: Chemical Applications of Synchrotron Radiation, Part I, ed. by Tsun-Kong Sham, Advanced Series in Physical Chemistry, Vol. 12A (World Scientific, 2002), p. 605.

4. F. J. Himpsel and K. N. Altmann, Electronic States of Magnetic Materials, Ch. 6, "Solid-State Photoemission and Related Methods: Theory and Experiment", W. Schattke and M. A. Van Hove (Eds.), Wiley-VCH, Berlin 2003, p. 177-219.

5. V. Pérez-Dieste, O. M. Castellini, J. N. Crain, M. A. Eriksson, A. Kirakosian, J.-L. Lin, J. L. McChesney, F. J. Himpsel, C. T. Black, and C. B. Murray, Thermal decomposition of surfactant coatings on Co and Ni nanocrystals, Appl. Phys. Lett. 83, 5053 (2003).

6. V. Perez-Dieste, J. N. Crain, A. Kirakosian, J. L. McChesney, E. Arenholz, A. T. Young, J. D. Denlinger, D. L. Ederer, T. A. Callcott, S.A. Lopez-Rivera, and F. J. Himpsel, Unoccupied orbitals of 3d transition metals in ZnS, Phys. Rev. B 70, 085205 (2004).

7. F. J. Himpsel, J. L. McChesney, J. N. Crain, A. Kirakosian, V. Pérez-Dieste, Nicholas L. Abbott, Yan-Yeung Luk, Paul F. Nealey, Dmitri Y. Petrovykh, Stepped Silicon Surfaces as Templates for One-Dimensional Nanostructures, J. Phys. Chem. B 108, 14484 (2004).

8. Fan Zheng, V. Pérez-Dieste, J. L. McChesney, Yan-Yeung Luk, Nicholas L. Abbott, and F. J. Himpsel, Detection and switching of the oxidation state of Fe in a selfassembled monolayer, Surf. Sci. 587, L191 (2005).

9. T. M. Schuler, R. A. Stern, R. McNorton, S. D. Willoughby, J. M. MacLaren, D. L. Ederer, V. Perez-Dieste, F. J. Himpsel, S.A. Lopez-Rivera, and T. A. Callcott, Electronic structure of the dilute magnetic semiconductor $\mathrm{Zn}_{0.90} \mathrm{Mn}_{0.10} \mathrm{~S}$ obtained by soft x-ray spectroscopy and first principles calculations, Phys. Rev. B 72, 045211 (2005).

10. D. Y. Petrovykh, V. Pérez-Dieste, A. Opdahl, H. Kimura-Suda, J. M. Sullivan, M. J. Tarlov, F. J. Himpsel, and L. J. Whitman, Nucleobase orientation and ordering in films of single-stranded DNA on gold, J. Am. Chem. Soc. 128, 2 (2006).

11. A. L. Schmitt, M. J. Bierman, D. Schmeisser, F. J. Himpsel, and Song Jin, Synthesis and properties of single-crystal FeSi nanowires, Nano Letters, in press (2006).

12. Xiaosong Liu, Chang-Hyun Jang, Fan Zheng, Astrid Jürgensen, J. D. Denlinger, Ronald T. Raines, Nicholas L. Abbott, and F. J. Himpsel, Characterization of RNase immobilization at Ag surfaces by NEXAFS, Langmuir, in press (2006).

13. P. Hoffmann, D. Schmeisser, H.-J. Engelmann, E. Zschech, H. Stegmann, F. Himpsel, and J. Denlinger, Characterization of chemical bonding in low-k dielectric materials for interconnect isolation: A XAS and EELS study, Mat. Res. Soc. Conf. Proc., in press (2006). 